\title{
Editorial \\ Catalysts: Special Issue on Plasma-Catalysis for Environmental and Energy-Related Applications
}

\author{
Monica Magureanu ${ }^{1, *}$ and Corina Bradu ${ }^{2, *(D)}$ \\ 1 Department of Plasma Physics and Nuclear Fusion, National Institute for Lasers, Plasma and Radiation \\ Physics, Atomistilor Street 409, 077125 Magurele, Romania \\ 2 PROTMED Research Centre, Department of Systems Ecology and Sustainability, University of Bucharest, \\ Spl. Independentei 91-95, 050095 Bucharest, Romania \\ * Correspondence: monimag@gmail.com (M.M.); corina.bradu@g.unibuc.ro (C.B.); Tel.: +40-766-457-701 (C.B.)
}

check for updates

Citation: Magureanu, M.; Bradu, C. Catalysts: Special Issue on Plasma-Catalysis for Environmental and Energy-Related Applications. Catalysts 2021, 11, 1439. https:// doi.org/10.3390/catal11121439

Received: 22 November 2021 Accepted: 24 November 2021 Published: 26 November 2021

Publisher's Note: MDPI stays neutral with regard to jurisdictional claims in published maps and institutional affiliations.

Copyright: (c) 2021 by the authors Licensee MDPI, Basel, Switzerland. This article is an open access article distributed under the terms and conditions of the Creative Commons Attribution (CC BY) license (https:// creativecommons.org/licenses/by/ $4.0 /)$.
Plasma-catalysis has been a topic of research for many years due to its potential for applications in a wide range of chemical, environmental, and energy-related processes. Non-thermal plasma offers an unconventional way to initiate chemical reactions in gas and in liquid due to the energetic electrons generated in the plasma, however, it suffers from low selectivity. The coupling of plasma with catalysis can steer the reactions in the desired direction, thus providing improved selectivity towards the target products and reducing unwanted ones.

This special issue demonstrates the interest in plasma catalysis as solution to environmental problems caused by greenhouses gases $\mathrm{CO}_{2}$ and $\mathrm{CH}_{4}[1,2]$ that can be converted to value-added products and fuels, gas pollution with stable polycyclic aromatic hydrocarbons [3] and volatile organic compounds [4], as well as water pollution [5]. The special issue includes a review article and four articles devoted to particular applications.

The review by Gao et al. [1] presents recent developments of plasma catalytic dry reforming of methane (DRM), focusing on dielectric barrier discharge (DBD) reactors in combination with nickel-based catalysts, currently considered as very promising catalysts in DRM reactions due to good catalytic activity, high availability and low cost. Results obtained using monometallic $\mathrm{Ni}$ catalysts on various supports $\left(\mathrm{SiO}_{2}, \mathrm{Al}_{2} \mathrm{O}_{3}, \mathrm{ZrO}_{2}\right.$ and activated carbon), as well as using Ni-based catalysts doped with other metals or compounds are summarized. The authors discuss the role of the support that can lead to different performance in the plasma catalyzed DRM reaction and present advantages and drawbacks of each support under plasma conditions, related to coke formation, metal sintering in connection with the size of Ni grains, dispersion of the Ni particles on the support, specific surface area in connection with reactant adsorption etc. Doping with other metals or metal oxides (alkali and alkaline earth metals- $\mathrm{K}$ and $\mathrm{Mg}$, transition metals-Co and $\mathrm{Mn}$, rare earth metals- $\mathrm{La}$ and $\mathrm{Ce}$ and their oxides) may prove beneficial, producing various effects such as enhancing resistance to carbon formation and even removing carbonaceous deposits, improving the $\mathrm{Ni}$ dispersion and inhibiting aggregation of Ni particles, strengthening the metal-support interaction, enhancing reactant adsorption, changing discharge characteristics as to increase the plasma area etc., ultimately leading to better plasma-catalyst synergy, higher $\mathrm{CO}_{2}$ and $\mathrm{CH}_{4}$ conversion and improved products distribution. Another important part of the paper is dedicated to plasma reactor design, including plasma-catalyst coupling, and process parameter optimization. The key parameters impacting on system performance and efficiency, i.e., discharge input power, feed flow rate, specific input energy, and feed ratio, are discussed in detail. The in-depth understanding of plasma-catalyst interaction and of the correlation between efficiency and reactor design and process parameters still remains one of the challenges to further improve this application.

Packing various materials inside the plasma reactor can influence the discharge in many different ways, obviously depending on the nature of the material, its dielectric 
constant, surface roughness, thermal and electrical properties, but also on their size and shape. Uytdenhouwen et al. [2] explored the potential of core-shell structured spheres to allow tuning of the packing properties for a specific application, by designing an optimal combination of core and shell. They investigated different core-shell combinations, using dense spheres of $\mathrm{SiO}_{2}, \mathrm{Al}_{2} \mathrm{O}_{3}$, and $\mathrm{BaTiO}_{3}$ as core and spray-coated powders of these materials as shell, and tested the materials for $\mathrm{CO}_{2}$ conversion in comparison with pure, dense spheres. The authors observed a clear effect of the size of the spheres on the conversion and energy efficiency, and the data showed that this effect is material-dependent. It was also found that the choice of core and shell materials is extremely important to the packed-bed reactor performance and further optimization can be carried out to some extent by adjusting shell thickness. Although it was not the purpose of this work to achieve high activity for $\mathrm{CO}_{2}$ splitting, as the tested materials are not catalytically active, nor to identify the optimum core-shell structure leading to significant performance improvement, the paper clearly demonstrates great potential in preparing packing materials appropriate for a certain application. Doping the shell with suitable catalysts, as suggested by the authors, represents a very interesting opportunity in this direction due to the macroporous nature of the material that may allow plasma to penetrate inside the pores and thus enhance plasma-catalyst interaction.

Various packing materials in a DBD reactor have also been investigated in the paper by Cimerman et al. [3] who reported the removal of tars, in particular naphthalene, by plasma catalysis in such a system. As previously mentioned, the packing can influence the discharge properties through several mechanisms, and this is ultimately visible in the variation of discharge power as a function of applied voltage. The authors focused on the effect of packing material on naphthalene removal and on the energy efficiency, considering the type of material, the specific surface area, the shape and size of the pellets. The pollutant removal was clearly enhanced when using the packed-bed reactor as compared to the empty DBD reactor, following the sequence $\mathrm{TiO}_{2}(88 \%)>\mathrm{Pt} / \gamma \mathrm{Al}_{2} \mathrm{O}_{3}(78 \%)>\mathrm{ZrO}_{2}(72 \%)>$ $\gamma \mathrm{Al}_{2} \mathrm{O}_{3}(66 \%)>$ glass beads $(64 \%)>\mathrm{BaTiO}_{3}(51 \%)>$ plasma only $(41 \%)$. It was suggested that a key factor for obtaining high removal efficiency is the presence of surface discharges that propagate along the packing material and thus activate a larger area of the catalyst and favor chemical reactions, while high dielectric constant materials, such as $\mathrm{BaTiO}_{3}$, concentrate the discharge close to the contact points of the pellets, resulting in weaker chemical effects. Increasing the specific surface area of the materials favored naphthalene removal, this behavior being similar to thermal catalysis, where textural properties play a major role. However, contrary to conventional catalysis, the shape and size of the packing material were found to be important in plasma catalysis, as they determine the size of voids between the pellets, and thus discharge properties, gas residence time and plasma volume. The authors identified carbon monoxide, carbon dioxide, water and formic acid as main products in the gas phase of naphthalene decomposition, as well as several other complex gaseous and solid by-products, indicating incomplete oxidation of the target compound.

Although recently significant research efforts have been devoted to explaining the mechanisms of plasma-catalyst interaction, the present understanding still leaves open questions related to the complex phenomena involved in plasma catalysis. In order to fill some of these gaps, the article by Vega-González et al. [4] focuses on the reaction pathways occurring during the decomposition of a volatile organic compound (acetaldehyde) by DBD fluidized bed with $\mathrm{Ag} / \mathrm{TiO}_{2}$ catalyst deposited on $150-\mu$ m-diameter $\mathrm{SiO}_{2}$ pellets. The authors obtained enhanced removal of the VOC and considerably higher selectivity to $\mathrm{CO}_{\mathrm{X}}\left(\mathrm{CO}+\mathrm{CO}_{2}\right)$ in the plasma-catalytic system as compared to plasma alone. For the highest specific input energy used (1150 J/L), acetaldehyde was almost completely removed $(98 \%)$ and the selectivity to $\mathrm{CO}_{\mathrm{X}}$ reached $60 \%$. Other organic by-products, such as methanol, acetic acid, methyl formate, methyl acetate, 1,2-ethanediol mono- and diformate, were identified in the gas phase in both reactors as a result of the target compound decomposition, while acetone and nitromethane were only detected in the plasma-catalytic process. In-situ diffuse-reflectance infrared Fourier transform spectroscopy (DRIFTS) was 
used to monitor the catalyst surface during acetaldehyde adsorption and subsequent plasma degradation. It was found that at the end of the adsorption step the $\mathrm{Ag} / \mathrm{TiO}_{2} / \mathrm{SiO}_{2}$ was mainly covered with acetaldehyde, but other species such as acetate, acetone and formaldehyde were also formed. The measurements carried out on the catalyst exposed to plasma reveal that acetaldehyde degradation proceeds with the formation of different surface compounds such as acetate, methoxy, ethoxy, formate and carbonate species, as well as formaldehyde and formic and acetic acid. In addition to the formation of these species on the catalyst surface, some of them can also be formed directly in the gas phase and subsequently adsorb on the catalyst. Detection of the carbonaceous intermediates provided more detailed information on acetaldehyde degradation in the plasma catalytic system by the interaction of adsorbed molecules with plasma-generated species and/or by-products of acetaldehyde decomposition in the gas phase. Thus, the main degradation pathways could be identified.

The degradation of aqueous pollutants is also a challenging issue that may be tackled by non-thermal plasma. Although the removal of target contaminants appears feasible, mineralization is often very slow and energy-consuming, as numerous organic by-products are formed, similarly to the case of gaseous pollutants. Again, the addition of catalysis is believed to provide a solution to this problem and various materials have been tested for this purpose in combination with plasma. Korichi et al. [5] addressed the issue of mineralization for a solution containing paracetamol treated by a DBD above liquid combined with iron catalysts supported on glass fiber and immersed in the liquid. Complete removal of the contaminant was achieved after $60 \mathrm{~min}$ treatment by plasma alone at very low input power $(0.3 \mathrm{~W})$, but the carbon remained in organic form and no mineralization was obtained. Introducing the $\mathrm{Fe}^{3+}$ catalyst proved beneficial to the degradation process: the rate constant increased by more than $40 \%$ as compared to the treatment by plasma alone, indicating faster paracetamol removal, and the energy yield was almost two times higher, clearly showing a considerable improvement in the process efficiency. Moreover, the mineralization degree increased to $30 \%$ after $15 \mathrm{~min}$ and to $54 \%$ after $60 \mathrm{~min}$ treatment by plasma catalysis, and a decreasing trend with treatment time was observed for most of the detected organic by-products. The role of the catalyst is thus decisive in improving the degradation process and was attributed to the generation of additional hydroxyl radicals by the Fenton-like reaction, i.e., by decomposition of the plasma-produced hydrogen peroxide in the presence of $\mathrm{Fe}^{3+}$. Hydroxyl radicals are highly reactive and generally believed to be the driver of the degradation of organic compounds in water in all advanced oxidation processes. Therefore, the plasma-Fenton combination, using the continuous supply of $\mathrm{H}_{2} \mathrm{O}_{2}$ generated in-situ by the plasma, shows great potential for water cleaning.

We can conclude that plasma catalysis is very active research area, various applications are envisaged and promising results are reported worldwide. Finally, we wish to thank all the authors for their valuable contributions, without which this special issue would not have been possible.

Conflicts of Interest: The authors declare no conflict of interest.

\section{References}

1. Gao, X.; Lin, Z.; Li, T.; Huang, L.; Zhang, J.; Askari, S.; Dewangan, N.; Jangam, A.; Kawi, S. Recent developments in dielectric barrier discharge plasma-assisted catalytic dry reforming of methane over Ni-based catalysts. Catalysts 2021, 11, 455. [CrossRef]

2. Uytdenhouwen, Y.; Meynen, V.; Cool, P.; Bogaerts, A. The potential use of core-shell structured spheres in a packed-bed DBD plasma reactor for $\mathrm{CO}_{2}$ conversion. Catalysts 2020, 10, 530. [CrossRef]

3. Cimerman, R.; Cíbiková, M.; Satrapinskyy, L.; Hensel, K. The effect of packing material properties on tars removal by plasma catalysis. Catalysts 2020, 10, 1476. [CrossRef]

4. Vega-González, X.; Duten, S. Sauce, Plasma-catalysis for volatile organic compounds decomposition: Complexity of the reaction pathways during acetaldehyde removal. Catalysts 2020, 10, 1146. [CrossRef]

5. Korichi, N.; Aubry, O.; Rabat, H.; Cagnon, B.; Hong, D. Paracetamol degradation by catalyst enhanced non-thermal plasma process for a drastic increase in the mineralization rate. Catalysts 2020, 10, 959. [CrossRef] 